\title{
Modular Integrated Electronic Experiment Testbed for Signal Processing Education
}

\author{
Zhang Yu-Xi, Wang Zhe, and Wang Jun
}

\begin{abstract}
With the rapid development of informatization, there is an increasing demand for electronic information talents, as well as higher requirement for their comprehensive quality. To meet the needs and combine with the distinctive domain of Beihang University in aeronautics and astronautics, this paper presents a physical wireless communication testbed which is not only a hybrid of communication, radar and navigation majors, but can also intend to be applied as an educational resource in core curriculums of electronic engineering and develops to an all-in-one experimental platform. This testbed advances the educational opportunities available to the audio wireless communication and basic protocols involved in signal transmission by providing a "real-world" platform where students can master signal processing and transmission methods from the perspective of engineering application and establish an integrated system of knowledge. It cascades almost all undergraduate courses and performs like a fundamental, comprehensive and innovative compound platform which can arouse students' interest, improve their professional practice and cultivate their innovation ability in a better way.
\end{abstract}

Index Terms-Audio communication, modular design, testbed, experimental teaching.

\section{INTRODUCTION}

With the rapid development of informatization, there is an increasing demand for electronic information talents, as well as higher requirement for their comprehensive quality. They should not only have solid theoretical foundation, but also capability of engineering practice. In undergraduate teaching system, courses including "Signals and Systems", "Discrete-time Signal Processing" and "Principle of Communication" are all basic and core curriculums for electronic information students. Helping them clarify main points of curriculum theory and master methods of engineering application are of great importance to their construction of knowledge hierarchy during undergraduate studies and also to their future study and career.

The common features of electronic information engineering courses are abstract concepts and complicated formula derivation, of which students may find it difficult to have a thorough understanding [1], [2]. On the other hand, the knowledge is always closely linked with engineering application, making students feel confused when apply protocols in "real-world". Therefore, as a beneficial supplement of higher learning, experimental teaching plays a significant role in developing and testing protocols and

Manuscript received April 15, 2016; revised June 17, 2016.

The authors are with the Electronic and Information Engineering Department, University of Beihang, China (e-mail: zhangyuxi@buaa.edu.cn wangz0424@163.com,wangj203@buaa.edu.cn). grasping the knowledge [3].

A common way for experimental teaching of electronic information engineering is implementing theories in simulators such as MATLAB or LabVIEW [4], [5]. This is commonplace in the educational community due to the flexibility and functionality of most popular programs who could also easily access to standard PCs [6]. In such cases simulating wireless communication and the following signal processing can be easily realized through writing codes or drugging components. However, in order to simulate a practical environment, there need modeling. In modeling, assumptions have to be made so as to simplify the "real-world". To get good modeling results, complex algorithms are applied because they contain more factors in wireless communication such as noise, clutter, multipath, and other limitation of communication. These above involve a number of complicated theoretical equations, which is far beyond undergraduate level. Even with a large amount of equations, this complicated modeling still fails to predict interferences in the real environment and to take all limits into consideration. In addition to the simplification of practical environment adopted by the simulations, the unintuitive of the physical layer modeling is also not closely linked with engineering application, resulting in an inevitable influence on the teaching quality. All these problems create the need of a physical platform that is capable of performing controlled experiments in a "real-world" environment in the educational community.

This "real-world" environment will mitigate the effects brought about by simplifications and provide more robust development. In addition to offering an environment where student can observe how protocols react in a real wireless environment, a physical platform is also able to improve on simulated environment at the same time. For example, instead of coding plenty of equations and complicated algorithms to simulate a real environment, both students and researchers can deploy the physical platform and get the data transmitted through real communication channel and then import them into MATLAB. In this way, they just need to focus on the subsequent data processing. This improves data authenticity and saves time that is valuable to fit the academic time schedule of experimental teaching [6].

Meanwhile, to meet the requirement of cultivating talents possessing the ability to solve complex engineering problems put forward by Ministry of Education, this paper advances the educational opportunities available to the audio wireless communication and basic protocols involved in signal transmission by providing a "real-world" platform. This testbed chooses audio which is familiar to students' daily life as transmit signals, aims at helping students gain a deeper 
understanding of the basic protocols involved in signal transmission, and master signal processing and transmission methods from the perspective of engineering application. Emulated experiments performed by the testbed are more targeted and more closely connected with engineering application then simulated experiments. Besides, they could also exercise students' practical ability, enhance their problem analyzing and solving abilities, therefore improve their comprehensive quality and motivate their innovation ability [7]-[9].

\section{AUdio WiRELESS COMMUNICATION TESTBED}

This paper has developed an audio wireless communication testbed that addresses the need for a scalable platform to be used as educational resource and research resource. The primary educational objective is to apply the testbed as an experimental teaching resource through its modular structure. By combining different modules together, faculties are capable of designing abundant experiments that cover undergraduate field of study.

In addition to education needs, the testbed also addresses the resource need for building a wireless environment that can supplement or replace simulation environment. The noise and interference available from the "real-world" make the data more authentic and reliable for the follow-up research.

Before detailing the description of hardware and software frame, the following subsections will begin with a brief overview of design concept of wireless communication system.

\section{A. Software Defined Radio Overview}

Software Defined Radios(SDR) was first proposed by Dr. Joseph Mitola III in May 1992, and with the development of communication engineering, there emerge many definitions. In this paper, SDR is a new radio system architecture and a design philosophy of modern radio engineering. Its basic concept is using scalable and reproducible hardware as a universal platform containing abundant radio functions which are realized by upgradeable and reconfigurable software [10]. Fig. 1 shows the schematic diagram of SDR structure.

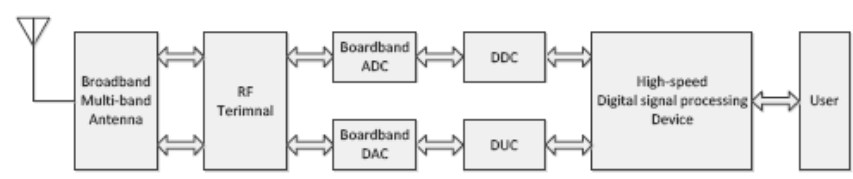

Fig. 1. Schematic diagram of SDR structure.

The audio wireless communication testbed can be divided into two major parts from its structure: transmitter and receiver. It is designed closely according with practical engineering and portable for experimental teaching. The functional module division of the system programming is beneficial for students to develop and test protocols and applications. Furthermore, as is common in daily life, audio signal has been chosen for transmission, in order to closely connect with the "real-world" and to stimulate students" interests and innovation.

\section{B. Testbed Hardware Structure}

The structure of audio wireless communication testbed is shown in Fig. 2. The testbed is mainly composed of three parts: peripheral circuit, FPGA, and RF circuit. Peripheral circuit is controlled by FPGA and is used for digital-analogue conversion. FPGA performs data encoding and decoding and digital up and down signal conversion. RF circuit converts IF signal to RF signal or vice versa, and finishes signal amplification and filtration processing at the same time.

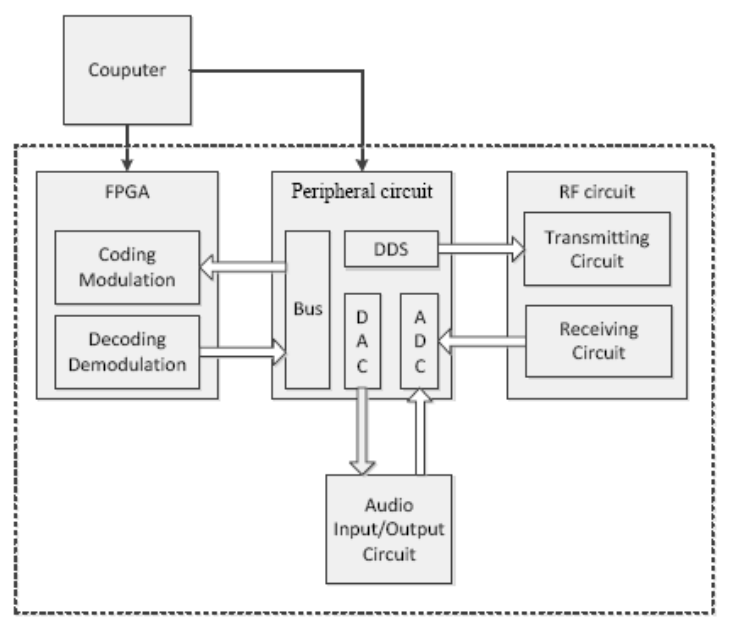

Fig. 2. Hardware structure of audio wireless communication testbed.

\section{Testbed Software Frame}

The software development environment is Xilinx ISE Design Suite and the programming language is VHDL. The framework of this software is based on SDR design philosophy and it divides communication system into signal sampling module, source coding module, digital modulation module, RF transmitting module, $\mathrm{RF}$ receiving module, digital demodulation module, source decoding module, and signal reconstruction module, as is shown in Fig. 3.

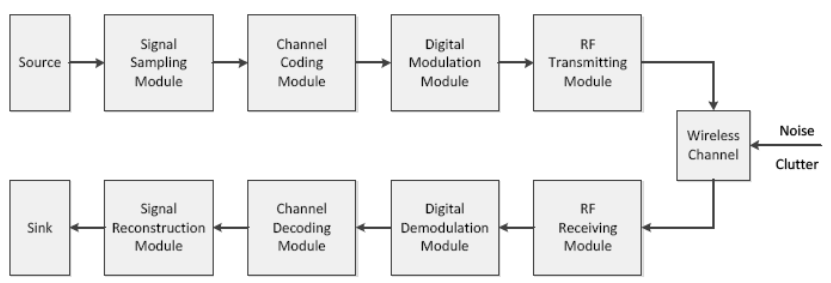

Fig. 3. Modular design of audio wireless communication testbed.

This modular design will not only improves the development efficiency, reduce cost, support subsequent function expansion, but also facilitate experimental teaching arrangement.

\section{MOdUlar EduCATIONAL COMPONENTS}

The primary task of this testbed is to be used as an experimental teaching platform. According to different modules and related theories, faculty can design plenty of verifications and extended experiments. They can use module individually to arrange experiments. For example, signal sampling-reconstruction modules are correlated to sampling and reconstruction of continuous-time signal in "Discrete-time Signal Processing" as is shown in Fig. 4. 


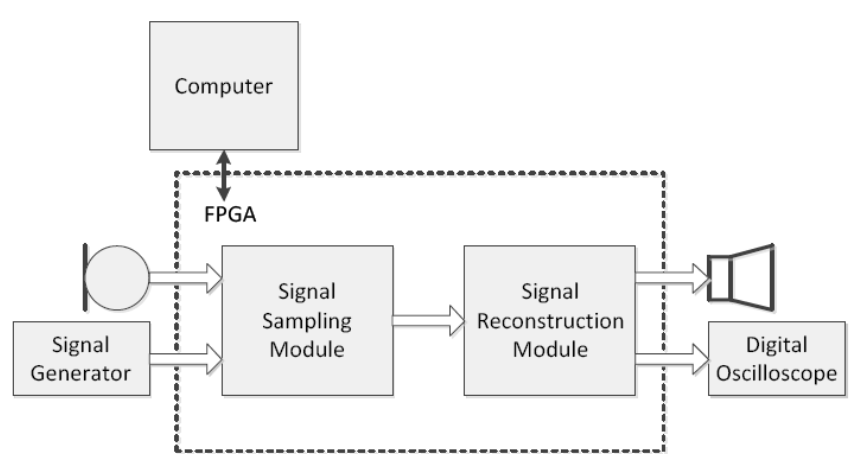

Fig. 4. Schematic diagram of sampling experiment.

During the arrangement of experiment, faculty could first let students sample a sinusoidal signal with a fixed frequency by using different types of sampling frequency to verify the Low-Pass Nyquist Sampling Theorem. With ChipScope or other EDA tools, students are able to observe the sampling points in a single cycle between under-sampled, critical sampling, and oversampling, and to compare the waveforms with theoretical derivation. Furthermore, by sampling the high-fidelity symphony and output through reconstruction module, they could also hear how different types of sampling frequency influence different tones. The same goes with sampling IF signal verifying Band-Pass Nyquist Sampling Theorem. In order to better understand the spectrum translation correlated with sampling process in frequency domain and mirror reactions within time domain and frequency domain, students can process the sampling signal on Fourier transform through FFT Core in FPGA.

Beside, faculty can also combine multiple modules together. For instance they can connect signal sampling-reconstruction modules with digital modulation-demodulation modules on the basis of analog linear modulation, analog angle modulation, digital carrier transmission and pulse code modulation in "Principle of Communication". Take the voice transmission that is based on 2FSK modulation as an example, and the module structure is shown in Fig. 5.

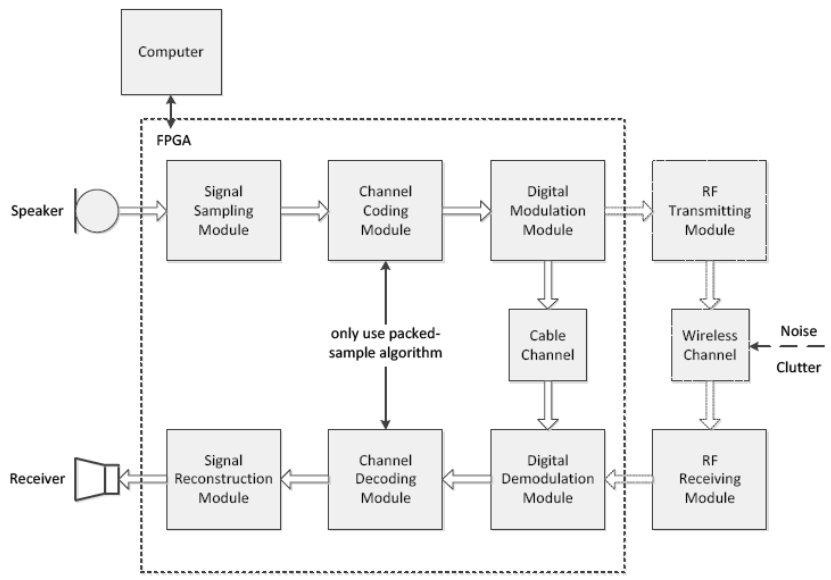

Fig. 5. Schematic diagram of voice transmission experiment.

In this experiment, a speaker converts his voice signal into digital signal in the signal sampling module first. Then the digital signal is modulated in 2FSK with selected frequency points and is then converted to IF signal from bass-band signal, a process that is implemented by digital modulation module. As for transmission mode, one can choose from wireless transmission, which needs RF transmitting-receiving module, and cable transmission, which is more simple and direct. After that, the receiver samples the IF signal through signal sampling module, and then finishes 2FSK demodulation by digital demodulation module and hence gets the bass-band signal. In this process, a variety of demodulation methods are available, including coherent demodulation, non-coherent demodulation, and zero-crossing detection. By comparing the complexity of algorithm and demodulation waveform with different demodulation methods, students are capable to summarize the BERs and applicable environment of each method. Finally, the voice signal is reconstructed by the signal reconstruction module. To alleviate workload, students can apply sample algorithm packed in channel coding-encoding module like channel coding-encoding, synchronization or any other signal processing methods that are involved in signal transmission.

With the modular structure, the testbed is applicable to most of undergraduate courses and makes experiment arrangement more flexible. This all-in-one experimental platform, based on a strong link with teaching materials, is more pertinent to the practical wireless communication system.

Apart from this, this testbed provides a wireless communication "playground" with plenty of resources for researchers to emulate theory and evaluate system in an unpredictable yet practical environment. Metaphorically speaking, this model is like a basin full of different Lego bricks. Faculty can creates some sort of structure by plugging different bricks together, referring to teaching materials, to design experiments. For a researcher, one can freely combine any blocks that he can find or even develop his own bricks.

\section{EXPERIMENTAL TEACHING IMPLEMENTATION}

At present, the experimental teachings of electronic information in engineering colleges is generally divided by subjects and relevant experiments in each subject are arranged without considering the inner relationship between subjects. Therefore, though, it could help students develop and test the knowledge they have learnt in class to some extent, it lacks of comprehensiveness as well as strong links with engineering concepts unable to help students establish a complete set of concepts and ideas on application design.

The main feature of this all-in-one experimental teaching platform is that it contains and leverages all different levels of undergraduate students. On the basis of the educational teaching system and according to characteristics of the curriculums, this paper presents a three-level teaching program by assembling the testbed: Verification-Driven experiment, Design-Driven experiment, and Innovation-Driven experiment.

Unlike the experimental teaching that is arranged and divided by subjects in most engineering colleges, this testbed, oriented in fulfilling wireless communication, arranges experiments by modular design philosophy, emphasizes connecting and integrating knowledge in different curriculums together and attaches great importance to applying theoretical knowledge in solving practical engineering problems. 
Table I summarizes the different kinds of experiments that the audio wireless communication testbed will provide.

TABLE I: TEACHING PROGRAM LEVEL DESCRIPTIONS

\begin{tabular}{lcl}
\hline \hline Level & \multicolumn{1}{c}{ Category } & \multicolumn{1}{c}{ Description } \\
\hline Low & Verification-Driven & $\begin{array}{l}\text { Simple use packed-sample } \\
\text { algorithms and plug pre-defined } \\
\text { module } \\
\text { Complete or rewrite the code on } \\
\text { the basis of practical technique } \\
\text { specification }\end{array}$ \\
High & Design-Driven & $\begin{array}{l}\text { Freely combine any modules } \\
\text { and create one's own system }\end{array}$ \\
\hline \hline
\end{tabular}

\section{A. Verification-Driven Experiments}

Verification-Driven experiments provide students an opportunity to use packed-sample algorithms according to the basic concepts and protocols they have learnt in class and allow them to observe signal transmission process between each module of the testbed and internal signal waveform before analyzing its spectrum. As a "physical exhibition" of relevant theories, this kind of experiments specify the abstract content and simplify the procedures of verifying and testing concepts and protocols. Such as Sampling Experiment mentioned above and Fig. 6 plots the sampling waveform.

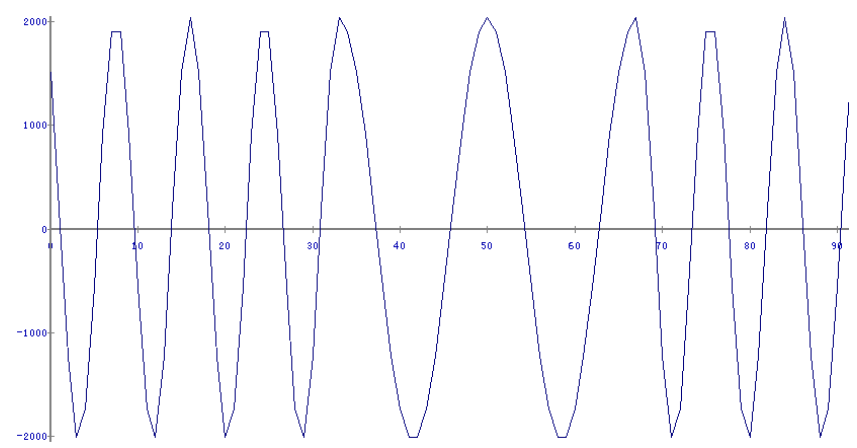

Fig. 6. Sampling waveform from sampling experiment.

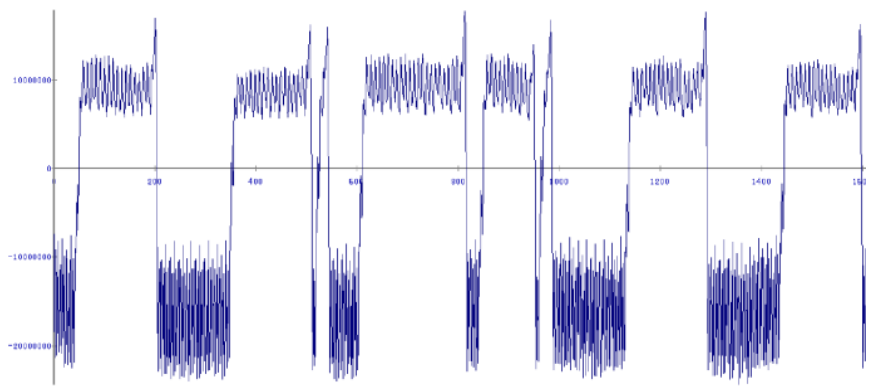

Fig. 7. Demodulation waveform from 2FSK communication experiment.

\section{B. Design-Driven Experiment}

Design-Driven experiments offers part of core codes of chosen modules, enabling students to complete or rewrite codes that would meet the practical technical specification. On one hand, these experiments can deepen students' understanding of signal processing and hardware emulation. On the other hand, they can develop students' ability of manual dexterity and innovation. By using modules designed by themselves instead of the existing modules, students can test the correctness of algorithm and achieve wireless communication. For example, 2FSK Communication Experiment which uses signal sampling-reconstruction modules and digital modulation-demodulation modules, and the demodulation waveform is plotted in Fig. 7.

\section{Innovation-Driven Experiment}

Innovation-Driven experiments give students a chance to play their creative freedom as long as they guarantee the achievement of wireless communication. According to the theories in teaching materials and methods of software design in the literature, they can choose their own way of coding-decoding, modulation-demodulation, etc. Apart from this, they can use existing modules flexibly to complete their systemic learning of wireless communication, to exercise professional practice and to cultivate innovation ability.

\section{CONCLUSION}

In line with strong theoretical and practical characteristics of core curriculums of the electronic information major, this paper advances a teaching method suggesting that practical engineering be taken as a starting point, integrating theoretical knowledge, and arranging experiments in different levels. This audio wireless communication testbed, based on a modular design method, cascades almost all undergraduate courses and performs like a fundamental, comprehensive and innovative compound platform which can arouse students' interest, improve their professional practice and cultivate their innovation ability in a better way.

This all-in-one platform satisfies the need of a testing and development "playground" for both undergraduate students and researchers. Its scalable and reconfigurable characteristics make it suitable for multi-level users to approach and design their own systems.

\section{ACKNOWLEDGMENT}

Wang Zhe thanks Wang Jun-Kai, Wang Zhan-Chao, Yang bin for the guidance given in designing this testbed. And also thanks Zhao Dan-Yu for the help of writing this paper.

\section{REFERENCES}

[1] Q. Wang and H. Yuan, "Exploration and practice of "Digital Siganl Processing" teaching method," Journal of Electrical \& Electrical Education, vol. 30, no. 8, pp. 87-89, 2008.

[2] J. Zhu, Z. Zhang, and Z. Zhu, "Research and practice on experimental teaching of "Digital Signal Processing"," Research and Exploration in Laboratory, vol. 27, no. 5, pp. 96-98, 2008.

[3] Z. Yang, X. Peng, and Y. Yu, "Research on practical teaching method of "Digital Signal Processing"," Research and Exploration in Laboratory, vol. 33, no. 9, pp. 181-183, 2014.

[4] Y. Shen, "Comprehensive experimental design of "Digital Signal Processing" based on MATLAB," Research and Exploration in Laboratory, vol. 28, no. 8, pp. 60-62, 2009.

[5] L. Chen, Y. Liu, and L. Qin, "Design of "Digital Signal Processing" experimental platform based on LabVIEW," Journal of Jilin Institute of Chemical Technology, vol. 28, no. 11, pp. 66-69, 2011.

[6] T. R. Newman and T. Bose, "Design of "A cognitive radio network testbed for wireless communication and signal processing education," presented at the Digital Signal Processing Workshop and $5^{\text {th }}$ IEEE Signal Processing Education Workshop, 2009. DSP/SPE 2009, IEEE $13^{\text {th }}$. pp. 757-761, 2009.

[7] J. Lin, "Research on professional training program of "Outstanding Engineer Education and Training Program"," Tsinghua Journal of Education, vol. 2, pp. 47-55, 2011.

[8] H. Liu, L. Zeng, and D. Zeng, "Research on the practice teaching system of electrical and electronics for "Outstanding Engineer 
Education and Training Program"," Research and Exploration in Laboratory, vol. 31, no. 7, pp. 358-360, 2011.

[9] Z. Sun, C. Chen, and S. Zhong, "Enterprise engineering practice training mode for the outstanding engineer," Research and Exploration in Laboratory, vol. 31, no. 8, pp. 285-288, 2012.

[10] C. Lou, J. Xu, and X. Yang, Software-Defined Radio: Principles and Practice, 2nd ed. Beijing, China: Publishing House of Electronics Industry, 2014, ch. 1, pp. 7-8

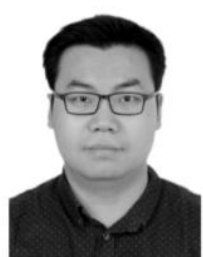

Yu-Xi Zhang was born in Henan, China, in 1985 and received Ph.D. degree from Beihang University, Beijing, China, in 2012.

$\mathrm{He}$ is currently an experiment teacher in the School of Electronic and Information Engineering, Beihang University. His researches include development of virtual-actual combination experiment platform virtual simulation experiment teaching, parallel signal real-time processing and embedded system of DSP/FPGA.

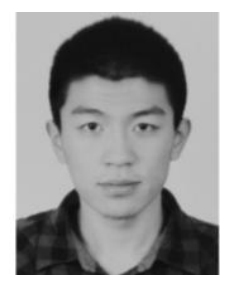

Zhe Wang received the B.S. degree in electronic and information engineering from Beihang University, Beijing, China, in 2015, where he is currently working toward M.S. degree in signal and information processing.

His currently research interests include signal processing, wireless communication system, MIMO and FPGA applications.

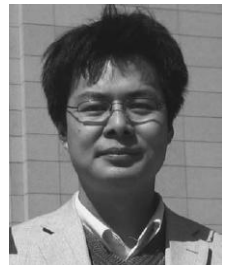

Jun Wang received the B.S. degree from the Northwestern Polytechnical University, Xi'an, China, in 1995 and the M.S. and Ph.D. degrees from the Beihang University, Beijing, China, in 1998 and 2001, respectively.

$\mathrm{He}$ is currently a professor with the School of Electronic and Information Engineering, Beihang University. He is interested in signal processing,

DSP/FPGA real-time architecture, target recognition and tracking, and so on. His research has resulted in 38 papers in journals, books, and conference proceedings. 